\title{
Gibsonian Information: an agent-based paradigm for quantitative information
}

\author{
Bradly Alicea ${ }^{1,2}$, Daniela Cialfi ${ }^{1,3}$, Avery Lim ${ }^{1,4}$, and Jesse Parent ${ }^{1}$ \\ Keywords: Ecological Psychology, Information Theory, Development and Plasticity, \\ Naturalistic Perception
}

\begin{abstract}
We propose a new way to quantitatively characterize information: Gibsonian Information (GI). This framework is relevant to both the study of cognitive agents and single cell systems that exhibit cognitive behaviors. Gl provides a means to characterize how agents extract information from direct perceptual signals. This differs from existing information theories in two ways. The first involves an emphasis on sensory processing, engagement in collective behaviors, and the dynamic evolution of such interactions. GI is useful for understanding first-order sensory inputs in terms of agent interactions with naturalistic contexts and higher-order representations. This allows us to extend $\mathrm{GI}$ to cybernetic and other types of symbolic systems representations. GI also emphasizes the role of information content in the relationship between ecology and nervous systems. Along with direct sensory input and simple internal representations, statistical affordances (clustered information that is spatiotemporally dependent perceptual input) facilitate extraction of $\mathrm{Gl}$ from the environment. As a quantitative accounting of perceptual information, $\mathrm{Gl}$ provides a means to measure a generalized indicator of nervous system input, and can be characterized by three scenarios: disjoint distributions, contingent action, and coherent movement. All of these cases provide a means to create a differential system between both motion (information) and random noise/stasis (non-information). By applying this framework to a variety of specific contexts, including a four-channel model of multisensory embodiment, we demonstrate how $\mathrm{Gl}$ is essential to understanding the full scope of cognitive information processing.
\end{abstract}

\section{Introduction}

Information can be difficult to measure and define. With respect to cognition and the brain, information is a polysemic concept (Floridi, 2011) that can be formalized in a number of different ways: from Shannon Information (Shannon et.al, 1949), Bayesian Surprise (Itti and Baldi, 2009), and even Integrated Information Theory (Oizumi et.al, 2014). We will introduce an alternative way to measure and otherwise characterize information content based on direct perception and ecological perspectives on

\footnotetext{
1 Orthogonal Research and Education Laboratory, Champaign-Urbana, IL and Worldwide bradly.alicea@outlook.com

${ }^{2}$ OpenWorm Foundation, Boston, MA

${ }^{3}$ University of Chieti-Pescara, Pescara Italy, danielacialfi@gmail.com

${ }^{4}$ Center for Enabling EA Research and Learning, Blackpool, UK
} 
perception. This perspective allows us to emphasize the nature of information as a continuous dynamical phenomenon, dependent on both an embodied observer and multiple sensory modalities. In particular, we can contrast our perspective of information in cognitive and living systems with Shannon Information, as both perspectives have broad theoretical and practical application. Additionally, there are two properties which make our alternative perspective unique: considering the role of an observer, and embodiment of the perceptual agent. The observer has a particular and important rule regarding how the information is measured: the measurement of the spatial and temporal frame of one observer can be different from that of another agent. Embodiment is also critical to this perspective, and while our approach can be applied to many different types of agent, from biological cells to multicellular organisms with nervous systems, and from computational agents to multi-agent collectives.

Gibsonian Information (GI), inspired by the work of Ecological Psychologist J.J. Gibson (Gibson, 1979), provides us with a measurement for information both intrinsically tied to embodied agents. Defining information in the ecological sense (GI) is much different than a purely statistical or algorithmic one. GI relies on objects that are subject to apparent motion as an agent moves through and changes perspectives on its environment. These objects can be considered affordances (Chemero, 2010), the information for which is contained in events that result in conjunction with agent interactions. These events occur in both time and space, and occur both externally and internally to the agent (Gibson, 2000). Gl also provides us with possibilities of action given the apparent movement of objects relative to agent interactions (Rietveld and Kiverstein, 2014). This provides us with a generative model of information that allows for information to be extracted from time-dependent, collective behavioral, and statistically abnormal contexts.

GI provides both a unique interpretation of Shannon Information in addition to an alternative framework that stresses the observer-centric and affordance-based aspects of perceptual information. There are seemingly superficial but critical differences between the two frameworks. Whereas Shannon information relies upon the presence of variation, GI instead relies upon the presence of interpretable motion against a background. The relationship between motion and background are potentially differentiable in a number of ways: the mode of difference is left up to the individual observer. From the perspective of information processing in agents, this can be summarized as direct perception that is detectable in systems as diverse as seeing biological motion in humans (e.g. Johanssen, 1973) and electroreception of prey in sharks (e.g. Hopkins, 2010). Yet the non-reliance of GI on internal representations may even allow us a mechanism for grounding semantic content (Harnad, 1990) in interactions with affordances and their spatiotemporally dependent context. 
One core notion in $\mathrm{Gl}$ is that information arises from inhomogeneous information and motion in the environment. This differs from Shannon Information, where information content is synonymous with diversity and unpredictability (Dretske, 1981). In particular, the ability of an observer to detect motion against a static background and to exploit spatiotemporal distinctions in environmental information are key to the utilization of Gl. The combination of an embodied context in which there are multiple modes of sensory input and interpretable motion provides affordances that evoke structure in the environment. While we stop short of proposing a mathematical theory of affordances, our mathematical formulation of $\mathrm{GI}$ relies on the structure provided by affordances as an external source of information that is encoded into the nervous system through perceptual processes.

In a manner similar to Shannon Information, we can define $\mathrm{Gl}$ in terms of an ensemble. A GI ensemble is a sequence of events observed in a spatial array. This results in a distribution that is not binomial: in fact, any number of continuous distributions. We can think of a movement information signal with rich spatial structure as an Exponential distribution (Power or Poisson) and random background noise as a Gaussian distribution. This is similar, at least in principle, to the concept of visual momentum (Woods, 1984). Visual momentum is the reinforcement of relevant data to support an effective distribution of direct perceptual information, allowing an agent to extract and integrate information over time, and is driven by spatial parallelism. This is related to reentrance in the brain and the internal communication channel of a $\mathrm{Gl}$ representation. Athalye et.al (2018) have observed that neural activity patterns leading to the reinforcement of environmental structure receive more frequent feedback. High visual momentum results from a continuity of action, while low levels result from discontinuous action. As we will see later this is also a key aspect of Gl. The relationship between perceptual motion and spatiotemporal distributions yield an information measurement that is captured in terms of a parameter value or a scalar representing the degree of informational change from one time point to the next. This provides us with a dynamical behavior rather than a discrete measure of bits.

Additional linkages between Shannon and Gibsonian Information come in their relationship to entropy. In the case of GI, Swenson and Turvey (1991) point out that sensation provides a basis for action and movement, which in turn drives entropy production. As a feedback loop, the thermodynamics of sustained movement are both constrained and driven by entropy production. Direct perception serves to shape the internal representation as influenced by the energetic constraints of temporal updating and behavioral effects of the agent on that updated information. Shannon Information has been shown to have a correspondence to thermodynamic entropy as far back as 
Landauer (1961), who showed that information processing requires physical entropy to be produced. This suggests a significant role for external information in shaping the information content of agent perception.

GI may also be important in the study of non-neuronal systems as found in slime molds (Boisseau et.al, 2016), the ciliate protozoan Stentor (Dexter et.al, 2019), and any number of unicellular organisms (Reina et.al, 2018). Gl is particularly compatible with an understanding of such systems, as Ecological Psychology does not presume any specific type of internal representation or computational mechanism (Fultot et.al, 2019). These systems, characterised by both the absence of a central nervous system and the presence of a body (cell), demonstrates the essential role of embodied phenotypes for functions that are analogous to information processing and even decision-making processes. These types of systems operate on information relevant to both individuals and colonial groups. Overall, GI is broadly applicable to the sensory worlds of individuals and social groups. The influence of $\mathrm{Gl}$ on social groups may also include the ability of agents to interpret their place in collectives such as schools, herds, and flocks (Toner and Tu, 1998).

\section{Gibsonian Information: mathematical definitions}

$\mathrm{Gl}$ is defined by motion, or more generally the distribution of objects in space and time. Unlike Shannon Information, $\mathrm{Gl}$ is defined by the arrival of environmental information in space and time. Ideally, this is described statistically in the form of a Poisson distribution. This suggests parallels between $\mathrm{Gl}$ and Fisher Information (Madiman et.al, 2007). The statistical signature of $\mathrm{Gl}$ is embedded in a spatiotemporally- dependent external communication channel (Figure 1) which delivers sensory input to an agent's sensory apparatus. The external and internal communication channels which define $\mathrm{Gl}$ and its internal representation are described mathematically in the form

$$
\begin{gathered}
g i(t)=\frac{\lambda^{x}}{x !} e^{-\lambda} \\
g i(t)^{\prime}=\frac{\lambda^{x}}{x !} e^{-\lambda}+\tau
\end{gathered}
$$

where $i(t)$ is the information function over time, $\tau$ is temporal delay, and the channel content is defined by a standard Poisson distribution. 
The Poisson arrival model allows us to model the structure of the sensory environment as a set of discrete spatiotemporal points that turn on and off as the agent encounters clusters of objects called affordances. In GI, affordances enable an agent to distinguish between randomness and cognitive order. This could be a surface or signal that is distinct from a random background, or a surface or signal that has a characteristic orientation relative to the agent's position relative to the sensory input.

Our Poisson arrival model is an encoding of information that translates into the activity of the internal communication channel (Figure 1). Sensory signals enter the internal communication channel at the sensory apparatus via sensory transduction, which occurs at a characteristic rate. This rate is controlled by the parameter $\tau$, which can results in effects such as under- or over-sampling (e.g. flickering and aliasing). As GI relies upon feedback from previous states of the internal component, the temporal delay term $\tau$ can also be modeled as an evolution equation, which takes the form

$$
\tau^{\prime}=f(t, \tau)
$$

where the temporal delay evolves over time $t$. The ability to evolve the temporal delay as a feedback over time results in a mechanism for generativity, creating an internal representation that resembles an ever-changing and adaptive distortion of directly perceived sensory input.

The Poisson model of environmental information is only one possible way to represent an ensemble of sensory signals. In cases where an informative background must be separated from other relevant information, the following equation provides us with a differential measure of $\mathrm{Gl}$.

$$
g i(t)=\int_{0}^{n} d_{2}-d_{1}
$$

where $d_{i}$ is a statistical distribution characterizing each source of spatial information and $n$ is the extent of the contrast in spatial units. For a neutral background (one with no useful information), $d_{1}$ would be 0 .

Since $\mathrm{Gl}$ is dependent on the sensory organ, Gibsonian Information is often weighted according to a center-surround model of signal transduction. For example, signals encountered at the edge of a sensory field, or in an oblique manner, are down weighted as compared to signals encountered head-on in the middle of the sensory field. This can be represented in the internal communication channel as fuzzy or 
uncertain information. The internal communication channel (Figure 1) holds a representation of $\mathrm{Gl}$ as a encoded Poisson distribution with temporal transformation $(\tau)$. This representation can then be decoded as a means to drive behavior or other autonomous processes within the agent.

\section{Embodied Observers and Affordances}

One critical component of the $\mathrm{Gl}$ approach is viewing agents as embodied observers. Observers possess a body that embeds its information processing system, and interactions with the environment that provide an explicit point of view of the world. This point of view depends upon the relative congruence of sensory modalities, which further provides constraints due to the observer's location and prior experience. The relative congruence of sensory modalities are determined by how an observer leverages both isolated and correlative affordances (Chong and Proctor, 2020). Unlike Shannon Information, $\mathrm{Gl}$ is assumed to be content-free that is utilized in support of affordances (van Dijk et.al, 2015). While we ignore the role of attention in this paper, attention provides a generative mechanism for encoding information from the environment into the internal communication channel (see Figure 1).

Points of view are also critical to interpreting isolated affordances, which consist of objects such as geometric primitives, functional guideposts, or spatial wayfinding signals. In the context of $\mathrm{Gl}$, isolated affordances can be viewed as anomalies with respect to a random background, and serve to bootstrap the exploitation of $\mathrm{Gl}$ by an observer. Heft (1996) provides an explicitly spatial perspective on Gl. The action (movement in space) of an agent generates optical flow, which provides a continuous reciprocal interaction between the internal and external communication channels. Optical flow can be extended to flow in other sensory modalities as well: it is these multimodal, continuous aspects of the information that make GI distinct from other types of information measures.

Another important feature of $\mathrm{GI}$ is the utilization of what we call correlative affordances to integrate sensory inputs and make simple inferences. In the context of $\mathrm{GI}$, affordances are building blocks for environmental structure. Phenomena that co-occur or are repeatedly experienced in the environment are considered correlative affordances. One example of this co-occurrence is visual confirmation of a bouncing ball coupled with proprioceptive confirmation of the ball's gravitational force. While the minimal representation required to register a correlative affordance are two sensory channels and a small embodied neural network, correlative affordances can be a gateway to associative learning. 


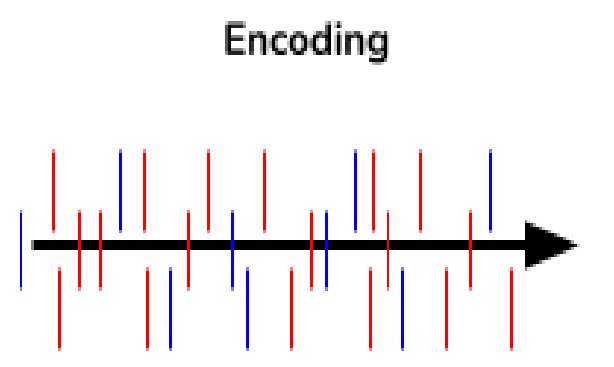

\section{External Communication Channel (spatiotemporally-dependent)}

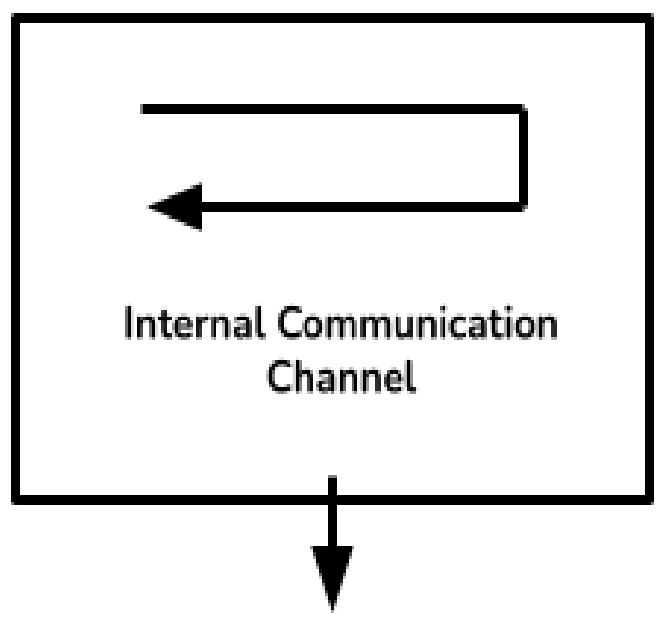

\section{Decoding}

Figure 1. Perception and processing of $\mathrm{Gl}$ in an agent. Shown are encoding and decoding steps, represented by the external environment and internal communication channel, respectively.

\section{GI as a Operational Phenomenon}

To better understand the Gl concept as an operational phenomenon, it is necessary to shed light on three important properties that lead us to the three quantitative principles of $\mathrm{Gl}$. These principles are characterized in terms of the disjoint distribution of sensation, contingent action and coherent movement (Figure 2). In addition, GI can also be observed by agents in terms of bars and gratings, as well as being captured by several parameters that can be contrasted with similar parameters defined for Shannon Information.

Disjoint distribution. The disjoint distribution of sensation is equivalent to disjoint information. While Mutual Shannon information (Ross, 2014) is defined as correlated information content between two categories, disjoint distribution is defined as the difference between two highly similar categories. Disjoint information allows us to distinguish two categories: one that is static and one that is dynamic. It is precisely this difference that leads to the measurement of GI: The greater the apparent motion in the dynamic series of images, the greater the GI. We can use white (or Gaussian) noise to demonstrate the role of disjoint distributions in GI. White noise serves as a means to activate brain mechanisms that allow us to interpret biological motion (Callan et.al, 2017). Our example in Figure $2 A$ shows a single frame of white noise static versus an animation of white noise static seen as a transition across multiple frames. As the difference between the two images, disjoint distributions reveal features such as the 
color state of specific pixel locations and motion cues. Disjoint distributions can be evaluated from both an egocentric and third-person perspective, and is best applied when a single observer is embedded in an environment separately from the influence of other observers.

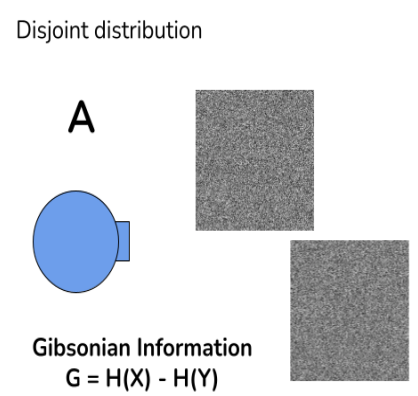
$G=H(X)-H(Y)$

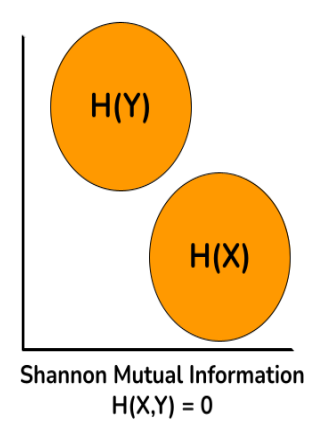

Coherent Movement

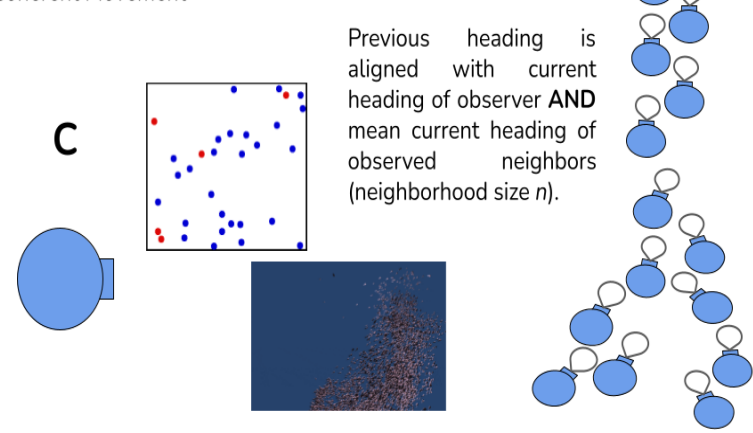

Contingent Action

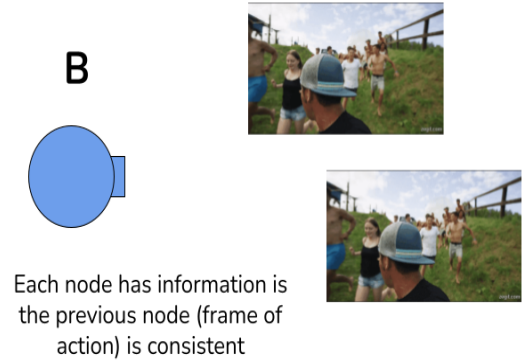

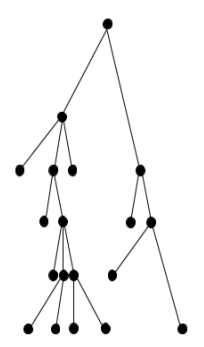

Figure 2. Three principles of $\mathrm{Gl}$, counterclockwise from top left: disjoint distribution (A), contingent action (B), and coherent movement (C).

Contingent Action. The second principle of $\mathrm{Gl}$ can be summarized as contingent action. When making comparisons between static and dynamic categories, dynamic categories are fundamentally different because they are subject to temporal contingency (Gallistel et.al, 2013): the sequence of images that make up the dynamic comparison in our disjoint distribution example exhibit a temporal causal dependence that can be modeled using a contingency tree. This dynamic comparison can be characterized as contingent action, and is shown in Figure 2B. The GI inherent in contingent action involves a comparison of two image sequences (animation), one with missing images in the sequence, and the other with the full sequence of images intact. This comparison should reveal that sequences with images removed from the periods of great configurational or contextual variation should yield relatively low amounts of $\mathrm{Gl}$.

In the example in Figure 2B, it might be enough to simply know that the Zorb ball is chasing the runners and what runners the ball rolls over. Conversely, images that contain great redundancy (conveying no transitional or configurational variation) can be removed without impacting the amount of GI. The sensitivity to configuration is 
comparable to Shannon Information, but Shannon Information does not explicitly deal with ordered motion as historical contingency. Long-term sensory augmentation can also result in contingencies which occur at a longer timescale (Konig et.al, 2016), but affect direct perception nonetheless.

Coherent Movement. Our final principle is called coherent movement. Returning to the disjoint distribution measure, we notice that while that measure makes a comparison between static and dynamic scenes, it says nothing about whether that motion characterizes coherent patterns such as biological or mechanical motion. In fact, our example in Figure 2A consists of visualized white noise. While disjoint distributions are passive in the sense that the observer need not be embedded in the flow of action, coherent movement itself is active and collective. Therefore, coherent movement is measured by considering the position of the observer during motion relative to other observers or objects in the environment.

In the example shown in Figure $2 \mathrm{C}$, the random motions of balls in a chamber is compared to flocking (Pulliam et.al, 1973; Maruyama et.al, 2019) and swarming (Bonabeau et.al, 1999; Kennedy and Eberhart, 2001) behaviors in virtual agents. In fact, direct perceptual processing from an egocentric perspective by each agent in a group may actually enable the type of flocking exhibited in a boid model (Reynolds, 1987; Ward et.al, 2001). Each agent in the flock (observer) evaluates their position (heading) with respect to their previous position AND mean current heading of all observer neighbors. The greater correspondence between the agent's expectation and the group behavior (or minimized divergence), the higher the Gl. Another example of coherent movement among individual agents is their observation of the environment around them. In the case of a shark, hydrodynamic phenomena (Zhou et.al, 2010) affect both sensation and the generation of movement through the environment. GI thus corresponds to laminar and turbulent flows, which in turn affect the sensation and movement against physical resistance (Wilga and Lauder, 2004).

\section{Gratings and Bars}

We can also use stimuli used in the study of visual perception as a means to construct toy model implementations of natural settings (Alicea and Gordon, 2014) captured by our three principles. For demonstration purposes, we will draw from the examples of gratings and bars. Colocating these two patterns against a neutral background provides affordances to the agent and increases the $\mathrm{GI}$ in its environment. In both of these cases, there is a high periodic structure unlike Gl found in a natural scene. Their input distribution resembles a periodic binomial distribution similar to systems with high Shannon Information. Natural scenes also have no clear background, which is especially true in the case of coherent movement. To make the connection 
between periodic patterns and attentional processes, stripes are represented in the brain in the form of ocular dominance columns (Hubel and Wiesel, 1962). This relatively simple neural representation is consistent with the internal communication channel shown in Figure 1, and has connections to preattentive processes and attentional enhancement (Grossberg, 2001).

To make our toy model example more interesting, we can use gratings of different frequencies for both the background and foreground. This allows for $\mathrm{Gl}$ to be evaluated as a set of differential distributions, where the background is subtracted from the foreground. In this case, environmental structure is represented by two competing distributions for which the differential strategy provides two possible evaluations of $\mathrm{GI}$ (for both foreground and background). Given a virtual reality stimulus, direct perceptual information viewed through the compound eye of the bee provides suppression of background and target cues enable discrimination learning, while background and target cues interact to affect associative learning (Lafon et.al, 2021).

\section{Potential Parameters for GI}

As is the case with Shannon Information, Gl can be distilled into a set of quantitative values that are tangentially related to the original measurement of information content. In the case of Shannon Information, $H$ can be distilled into $H_{\text {max }}$, $H_{\text {min }}$, and overall channel capacity. In Table 1, we show the differences between Shannon Information and GI in terms of various potential measures and their definitions.

\section{Four-channel Embodiment}

Now that we have an appreciation for $\mathrm{Gl}$ and its contrasts with Shannon Information, we can apply GI to a wider range of problems by using an example called four-channel embodiment. The existence of GI in embodied sensory channels can be demonstrated using the developmental Braitenberg Vehicle (dBV). dBVs (Dvoretskii et.al, 2020) allow us to consider direct perception in an embodied and developmental context where the neural network expands over time and can be modified by both innate mechanisms and sensory inputs. Standard Braitenberg Vehicles (Braitenberg, 1984) allow us to view direct perception from a quasi-anatomical standpoint: an internal architecture allows for behaviors to be produced from the direct perception of environmental cues such as inertial forces, light, or odorants.

Developmental versions of BVs allow us to examine the interaction between a changing internal architecture and direct perception. For a single sensory channel, we can observe various different architectural variations in response to these interactions with sensory information. These variations may prime these networks for later performance and learning benefits a phenomenon called developmental freedom 
(Alicea et.al, 2020). The addition of multiple sensory channels can add additional interactions to this basic framework, but with superadditive and suppressive effects. As both an acquisitional and morphological phenomenon, development is a key aspect of ecological approaches (Read and Szokolszky, 2018). In the case of dBVs, the developmental process provides insight into both how experience-dependent plasticity (Kleim and Jones, 2008) can enhance performance, and how different sensory cues in specific combinations can contribute to learning. These consequences are critical to understanding how $\mathrm{Gl}$ can enhance or (in some cases) enable inference of a sensory channel.

Table 1. Differences between Gibsonian and Shannon Information, and the method by which these differences might be measured.

\begin{tabular}{|c|c|c|c|}
\hline Gibsonian & Shannon & \\
\hline $\begin{array}{c}\text { Flow, movement, } \\
\text { contrast }\end{array}$ & $\begin{array}{c}\text { Maximum } \\
\text { coherent } \\
\text { movement } \\
\left(\text { MOV }_{\text {max }}\right)\end{array}$ & $\begin{array}{c}\text { Surprise, } \\
\text { heterogeneity, } \\
\text { diversity }\end{array}$ & $\begin{array}{c}\text { Maximum entropy } \\
\left(\mathrm{H}_{\text {max }}\right)\end{array}$ \\
\hline $\begin{array}{c}\text { Disjoint distribution } \\
\text { (difference between } \\
\text { moving pixels and } \\
\text { stationary pixels } \\
\text { over time) }\end{array}$ & $\begin{array}{c}\text { Minimum } \\
\text { coherent } \\
\text { movement } \\
\left(\text { MOV }_{\text {min }}\right)\end{array}$ & $\begin{array}{c}\text { Joint distribution } \\
\text { (mutual information } \\
\text { between pixels of } \\
\text { different states) }\end{array}$ & $\begin{array}{c}\text { Minimum entropy } \\
\left(\mathrm{H}_{\text {min }}\right)\end{array}$ \\
\hline $\begin{array}{c}\text { Temporally } \\
\text { dependent } \\
\text { (time-series) }\end{array}$ & $\begin{array}{c}\text { Flow Rate } \\
\text { Capacity }\end{array}$ & $\begin{array}{c}\text { ID (binomial } \\
\text { probability } \\
\text { distribution) }\end{array}$ & Channel Capacity \\
\hline
\end{tabular}

Now let us consider the idea of emergent direct perception as multisensory integration in the context of a biological nervous system. In this case, direct perception arises from both experience with the sensory information that constitutes a scene as well as the ability to integrate this information in a coherent manner. We can take as an example a human observer riding a roller coaster: An observer can see the track ahead of them, expecting visual movement in certain directions, but also experiencing strong inertial forces which contribute to the experience. Each sensory channel transmits a different sensory modality, and $\mathrm{Gl}$ is assessed by the degree of disjoint information 
between all channels. We expect to see a variety of superadditive and suppressive effects over time, particularly as cues of different modalities become incongruent.

\section{Multisensory Embodiment}

Given our interest in multisensory interactions, a four-channel analysis shown in Figure 3 serves as an instance of multisensory embodiment where the direction of information for a single static image (frame of an animation) points in three different directions. In the Figure 3 example, the rabbit is our observer and provides a means to embed multisensory $\mathrm{Gl}$ into a single set of external and internal signals.

As shown in Figure 3, each sensory channel has two possible signal types: direct perception and indirect perception. These signal types represent the external and internal communication channels, respectively. The signals themselves have a specific orientation and strength as shown by the direction and length of the arrow. When they are all pointed in the same direction, the sensory environment is said to be congruent. By contrast, signals that are pointed in different directions are incongruent, which has effects on multisensory integration and the overall coherence of a given percept. Examples of these signals providing dynamical information to the sensory channels can not only be shown for the rabbit in Figure 3, but also the examples in Figure 4. This provides a symbolic processing framework that is independent of the GI measurement schema shown in Figure 1.

From a broader perspective, there is an interesting relationship between disjoint distributions and multisensory embodiment of an agent that requires further study. In particular, the incongruence between stimuli shown in Figure 3 constitutes a disjoint distribution. Although all four sources fail to point in the same direction, this incongruence does not result in confusion or suppressive effects. Unlike the confirmatory action of congruent sensory channels, incongruence might lead to superadditive effects (e.g. Holmes and Spence, 2005) by serving as a counterfactual source of information (e.g. Kanai et.al, 2019) and a richer representation of the phenomenon in question. Indeed, our distinction between direct and indirect perception in Figure 2 is in line with this interpretation.

The roles of direct and indirect perception can also be demonstrated using an agent interacting with virtual reality. Virtual reality relies upon a computational simulation to create the illusion of a sensory environment. Once again, we can use our multisensory framework to understand what is going on in this interaction. Figure 4 demonstrates virtuality in the context of a human moving a virtual object with a wand and their upper body movements. In this case, a new form of direct perception called rotational momentum is introduced, present only in a single sensory modality. 

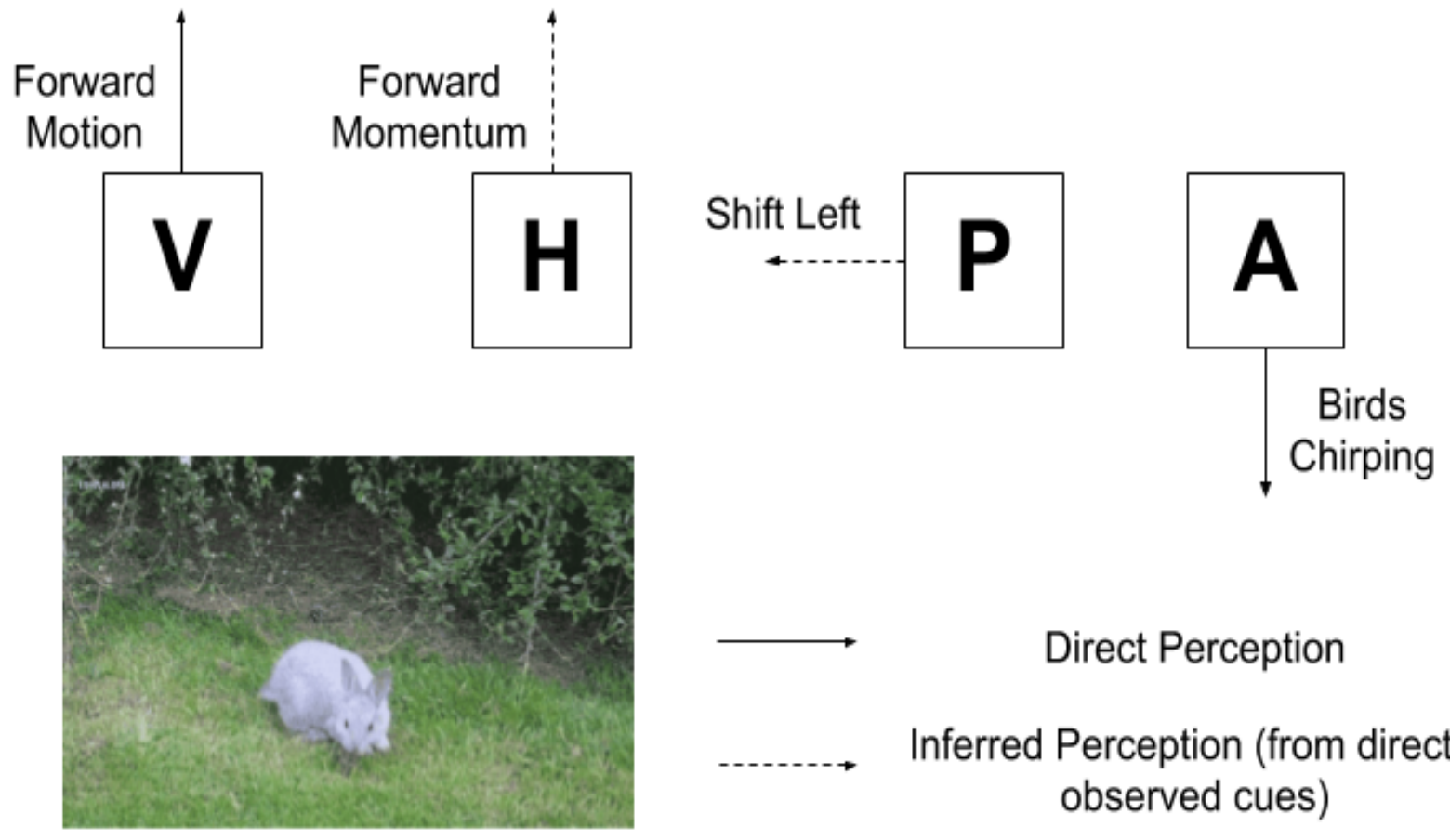

Figure 3. An example of four-channel embodiment: visual (V), haptic (touch - $H$ ), proprioception $(\mathrm{P})$, and audition $(\mathrm{A})$. The embodied observer is a rabbit hopping around a garden.

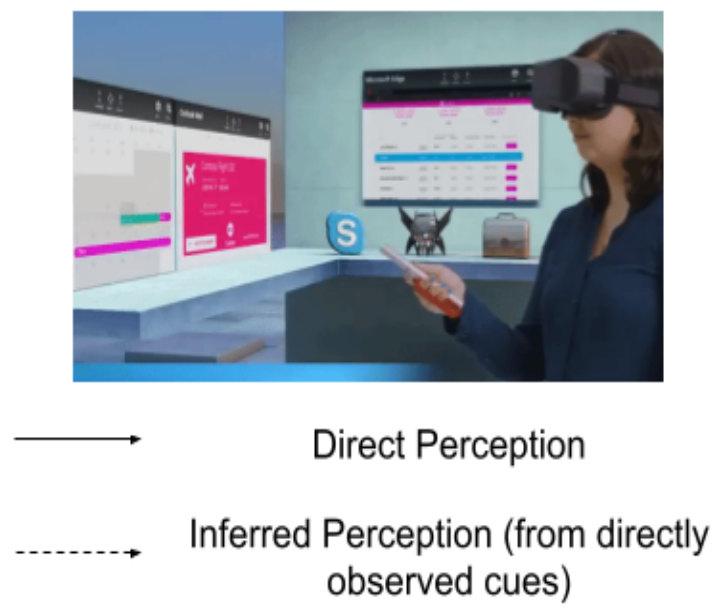

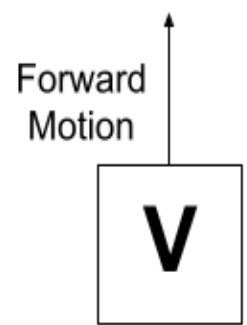
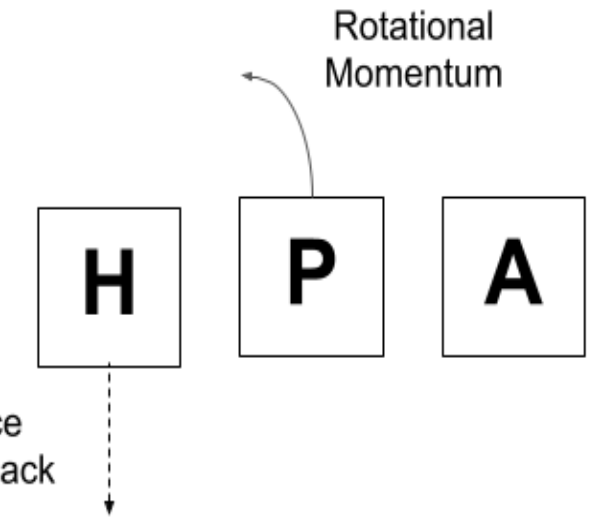

Figure 4. A secondary example of four-channel embodiment from human virtual environment navigation. Channels: visual $(V)$, haptic (touch $-H)$, proprioception $(P)$, and audition (A).

\section{GI in a Multi-sensor Array}

The original mathematical description of $\mathrm{Gl}$ assumed a single sensory apparatus, such as a single eye on an organism or a single haptic sensor on a robot. In most cases, $\mathrm{Gl}$ is captured by an array of multiple sensors and then integrated during 
sensory transduction. An example of this is shown in Figure 5. In the case of an unweighted single sensor, we can consider the internal representation in terms of 1-dimension of time and 1-dimension of space. This will result in a blinkering of state (on and off) as sensory points are encountered. The movement that defines $\mathrm{GI}$ will be smeared in time as spatial information will be lacking. With multiple sensors, we can use different integration strategies to weight the different contributions of spatial diversity in the stimulus, similar to the center-surround weighting described previously.

\section{GI in non-neuronal systems}

Extending our model to information processing systems without a neuronal network, Gl allows us to characterize both the internal and external communication channels of such a system. For example, in a Diatom cell, cells change their behavior with respect to light intensity (Cohn et.al, 2015). In bacteria, phototaxis and chemotaxis are the predominant mode of behavioral output (Ben-Jacob, 2008; Lan and Tu, 2016), and can be replicated using a small artificial neural network (Zhang et.al, 2021). These behaviors are driven by chemical and light gradients, respectively. GI involves both external and internal information processing mechanisms involving extracellular proteins and myosin fibers, respectively.

The motion of cells during migration and their arrangement in collectives are also an interesting application of $\mathrm{Gl}$. In this case, any single cell can exhibit collective motility during development. As part of a collective, this single cell can utilize coherent movement along with an integration of environmental cues as encoded input to estimate GI. GI is well suited to understanding the positional information cells use during development (Dubuis et.al, 2013; Tkacik and Gregor, 2021). In this case, the input is the distribution of gene expression values of a cell's biochemical milieu, and the model representing our internal communication channel is the mapping between the expected signal by location and the cell's autonomously-derived internal state.

\section{Discussion}

Now that we have proposed measures and observational principles of Gibsonian Information, particularly in contrast to Shannon Information, we can consider the concept of channel capacity (Cover and Thomas, 1991). Channel capacity is a core concept in Shannon Information and psychophysics alike, and is similarly important in Gibsonian Information. In Ecological Psychology (Gibson, 1979), implicitly defined capacities of sensory channels are used to enable an observer's ability to explore its environment (Lobo et.al, 2018). Channel capacity in the Gibsonian Information context is related to both cognitive capacity limits (Weems and Reggia, 2012) as well as transmission rate (Cover and Thomas, 1991). We can operationalize the dynamical 
channel capacity as a continuous flow of bits (represented as a time series) from the environment.

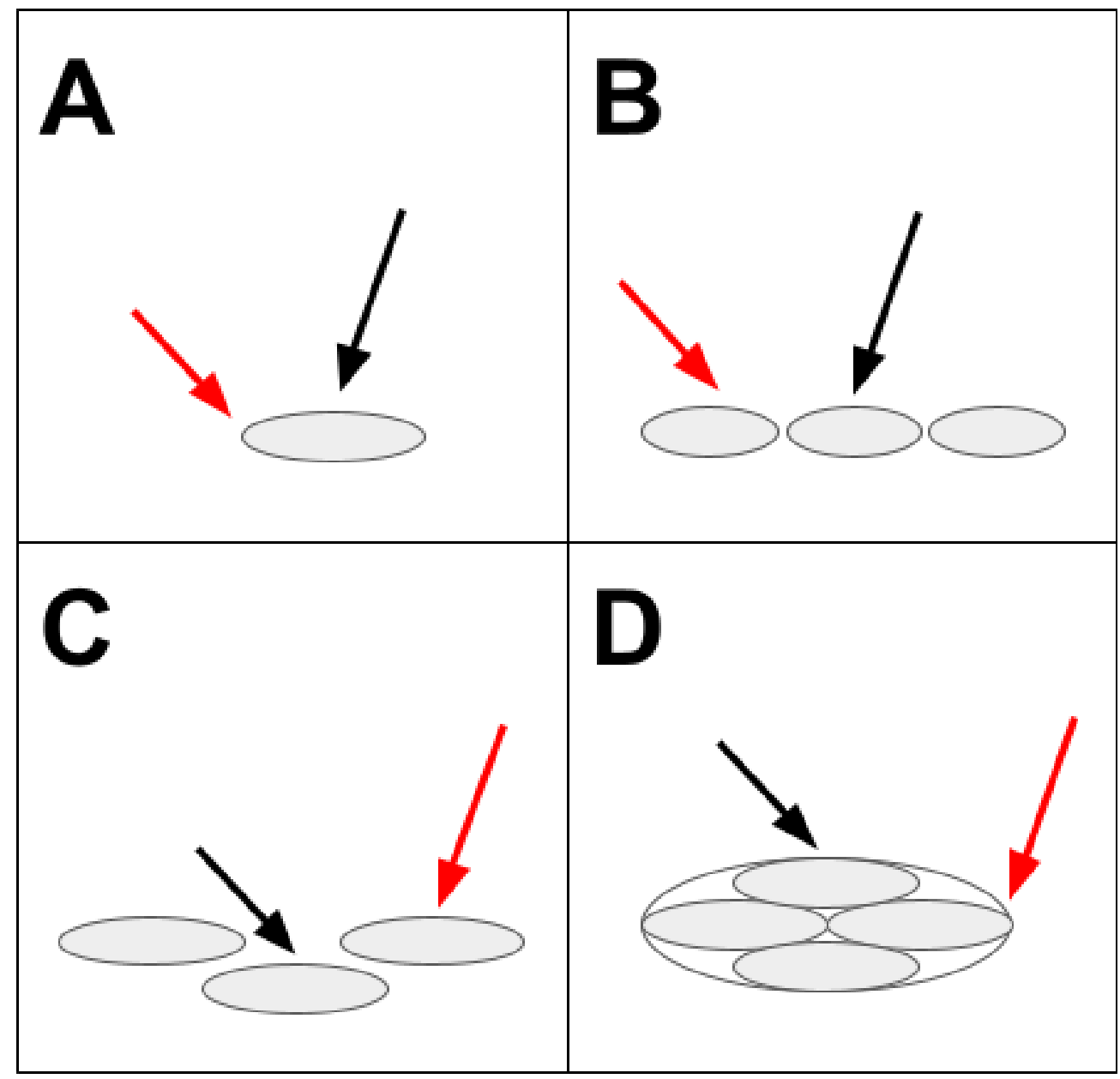

Figure 5. An agent that captures GI using a multi-sensor array and the resulting enrichment of spatial resolution. A: single sensor, B: parallel sensor array, C: semi-circular array, D: compound eye. Red arrow: input at edge of receptor field, Black arrow: input at center of receptor field.

\section{Overarching Themes and Future Directions}

Therefore, one overarching theme of $\mathrm{Gl}$ is the integration of sensory information, particularly over time. In biological observers, as opposed to computational agents, it is known that multisensory information is important in cognitive functions such as attention, and can arise from phenomena such as the observation of biological motion (e.g. Wu et.al, 2019) and latencies between different sensory modalities (e.g. Yu et.al, 2020). With respect to multisensory congruence then, future research should investigate the relationship between informational fluxes and changes in configurational diversity over time. As GI is an inherently dynamical approach to information, constructs related 
to the principles of disjoint distributions and coherent movement can capture these fluxes in structural information.

GI can be placed in a theoretical context of agent action such as Simultaneous Localization and Mapping (SLAM - Durrant-Whyte and Bailey, 2006) and the perception-action loop (Turvey, 2012). The basic SLAM algorithm decomposes perceptual input-output into four components: input, mapping, localization, and output. The internal components of the SLAM model provide spatial content, and map to the additive $\boldsymbol{\tau}$ term in Equation 2. As a delay parameter of the external encoding, in this case delay provides a means to model displacements in the representation of space. By contrast, the perception-action loop is based on an internal closed feedback loop that refines the external encoding using a Bayesian kernel or similar technique for statistical integration. Unlike with SLAM, our additive $\boldsymbol{\tau}$ term in Equation 2 represents temporal delay, and provides temporal context to the external signal. The application of $\mathrm{Gl}$ to a SLAM context is particularly useful in the case of collective behaviors (Kegeleirs et.al, 2021) and adds to our understanding of how Gl characterizes coherent movement.

When we consider a population of observers (agents), we can measure GI from different points of view in a common environment. This is potentially valuable for understanding the spatial variation and richness of features in a given environment. Every individual agent will take an egocentric view of their environment. In cases where a single agent has multiple sensory inputs (e.g. multimodal perception or a sensory receptor array), the egocentric viewpoint is enriched. Occasionally, an agent can take multiple views of the world if the sensor array covers different parts of the same body (e.g. head and tail). This fundamental difference between different viewpoints allows for a demonstration of disjoint distributions and the measurement of GI. Particularly, a mental frame of reference shared either socially or within a central nervous system must integrate disparate forms of information. This is much different than the ensemble approach of Shannon Information, where the variation between perspectives is condensed into a single parameter.

Returning to the connection between perceptual information and thermodynamics, the characterization of GI in dynamical systems suggests that rather than a closed-loop feedback system, informational regulation more resembles a futile cycle. Futile cycles are native to metabolic physiology (Qian and Beard, 2006), and consist of closed loop feedback processes where the only product is energy dissipation. This type of organization allows for robustness to large fluctuations in molecule number (or pieces of information). GI also allows us to consider the role of externalization in cognitive agents. Externalizing cognition (Clark and Chalmers, 1998; Bocanegra et.al, 2019) can be understood through the encoding and decoding components of the 
schema shown in Figure 1. Externalization means that more of the representation of information occurs prior to the encoding stage. Therefore, the input distributions will tend to have more structure, and the increased number of affordances will likely take the form of complex exponential distributions characterized in Equation 1.

\section{Relationship to Rival Approaches}

An alternative way to treat the probability distribution of environmental information is to map these points of information to a more general representation before encoding (see Figure 1). This allows us to build a mapping based on cybernetics and category theory (Cruttwell et.al, 2021). This spatially-explicit representation allows for the principle of coherent movement in space and contingent action in time. The former representation takes the form of a local-to-global map, and the latter is a tree-like structure. In terms of the resulting internal communication channel, we observe mappings between these representations that form a disjoint distribution. While the $\mathrm{GI}$ exists in these disjoint regions, there is also the potential for shared categories that also yield GI. Alternatively, there are connections between collective motion and non-classical logic (Krol et.al, 2021) that might serve to represent shared and disjoint categories in the internal communication channel. Such an approach might also allow us to extend the principle of disjoint categories in terms of category theory (Spivak, 2014).

Understanding direct perception as quantitative information may be quite effective for understanding phenomena like visual flow or in cases where the observers are slime molds and single cells. Yet in observers with complex nervous systems (such as bird flocks, hopping rabbits, or human roller coaster riders), direct perception may not be a sufficient means to explain how the sensory information translates into the inferential aspects of cognition and ultimately behavior (Fodor and Pylyshyn, 1981). Furthermore, direct perception is a non-representational view of the world, which limits its ability to identify and measure higher-level features of the environment (Hilario, 1997). This limits our ability to apply GI (at least in the form of quantitative measurements) directly to behavior. But we may also be able to apply $\mathrm{Gl}$ as the lower layer of a hybrid model of the observer in which $\mathrm{Gl}$ is used to inform and/or constrain model components with greater representational capacity (e.g. Fodor and Pylyshyn, 1988; Feldman and Ballard, 1982).

\section{References}

Alicea, B., Chakrabarty, R., Gopiswaminathan, A.V., Lim, A., Ozcelik, F., and Parent, J. (2020). Embodied Continual Learning Across Developmental Time Via Developmental Braitenberg Vehicles. arXiv, 2103.05753. 
Alicea, B. and Gordon, R. (2014). Toy models for macroevolutionary patterns and trends. BioSystems, 123, 54-66.

Athalye, V.R., Santos, F.J., Carmena, J.M., Costa, R.M. (2018). Evidence for a neural law of effect. Science, 359, 1024-1029.

Ben-Jacob, E. (2008). Social behavior of bacteria: from physics to complex organization. European Physical Journal B, 65(3), 315-322.

Bocanegra, B.R., Poletiek, F.H., Ftitache, B., and Clark, A. (2019). Intelligent problem-solvers externalize cognitive operations. Nature Human Behaviour, 3, 136-142.

Boisseau, R.P., Vogel, D., and Dussutour A. (2016). Habituation in non-neural organisms: evidence from slime moulds. Proceedings of the Royal Society B, 283, 20160446.

Bonabeau, E., Dorigo, M., and Theraulaz, G. (1999). Swarm Intelligence: From Natural to Artificial Systems. Oxford University Press, Oxford, UK.

Braitenberg, V. (1984). Vehicles: experiments in synthetic Psychology. MIT Press, Cambridge, MA.

Callan, A., Callan, D., and Ando, H. (2017). The Importance of Spatiotemporal Information in Biological Motion Perception: White Noise Presented with a Step-like Motion Activates the Biological Motion Area. Journal of Cognitive Neuroscience, 29(2), 277-285.

Chemero, A. (2010). An Outline of a Theory of Affordances. Ecological Psychology, 15(2), 181-195.

Chong, I. and Proctor, R.W. (2020). On the Evolution of a Radical Concept: Affordances According to Gibson and Their Subsequent Use and Development. Perspectives in Psychological Science, 15(1), 117-132.

Clark, A. and Chalmers, D. (1998). The Extended Mind. Analysis, 58(1), 7-19.

Cohn, S.A., Halpin, D., Hawley, N., Ismail, A., Kaplan, Z., Kordes, T., Kuhn, J., Macke, W., Marhaver, K., Ness, B., Olszewski, S., Pike, A., Rice, E., Sbarboro, J., Wolske, A., and Zapata, Y. (2015). Comparative analysis of light-stimulated motility responses in three diatom species. Diatom Research, 30, 213-225. 
Cover, T.M. and Thomas, J.A. (1991). Elements of Information Theory. Wiley, New York.

Cruttwell, G.S.H., Gavranovic, B., Ghani, N., Wilson, P., and Zanasi, F. (2021). Categorical Foundations of Gradient-Based Learning. arXiv, 2103.01931.

Dexter, J.P., Prabakaran, S., and Gunawardena, J. (2019). A Complex Hierarchy of Avoidance Behaviors in a Single-Cell Eukaryote. Current Biology, 29, 4323-4329.

Dretske, F. (1981). Knowledge and the Flow of Information. MIT Press, Cambridge, MA.

Dubuis, J.O., Tkacik, G., Wieschaus, E.F., Gregor, T., Bialek, W. (2013). Positional information, in bits. PNAS, 110(41), 16301-16308.

Durrant-Whyte, H. and Bailey, T. (2006). Simultaneous Localization and Mapping: Part I. IEEE Robotics and Automation Magazine, 13(2), 99-110.

Dvoretskii, S., Gong, Z., Gupta, A., Parent, J., and Alicea, B. (2020). Braitenberg Vehicles as Developmental Neurosimulation. arXiv, 2003.07689.

Feldman, J.A. and Ballard, D.H. (1982). Connectionist Models and Their Properties. Cognitive Science, 6, 205-254.

Floridi, L. (2011). The Philosophy of Information. Oxford University Press, Oxford, UK.

Fodor, J.A. and Pylyshyn, Z.W. (1981) How direct is visual perception? Some reflections on Gibson's ‘Ecological Approach'. Cognition, 9, 139-196.

Fodor, J.A. and Pylyshyn, Z.W. (1988). Connectionism and cognitive architecture: A critical analysis. Cognition, 28(1-2) 3-71.

Fultot, M., Frazier, P.A., Turvey, M.T., and Carello, C. (2019). What Are Nervous Systems For? Ecological Psychology, 31(3), 218-234.

Gallistel, C.R., Craig, A.R., and Shahan, T. (2013). Temporal Contingency. Behavior Processes, 101, doi:10.1016/j.beproc.2013.08.012.

Gibson, E.J. (2000). Where Is the Information for Affordances? Ecological Psychology, 12(1), 53-56. 
Gibson, J.J. (1979). The Ecological Approach to Visual Perception. Psychology Press, East Sussex, UK.

Grossberg, S. (2001). Linking the laminar circuits of visual cortex to visual perception: development, grouping, and attention. Neuroscience and Biobehavioral Reviews, 25(6), 513-526.

Harnad, S. (1990) The Symbol Grounding Problem. Physica D, 42, 335-346.

Heft, H. (1996). The ecological approach to navigation: a Gibsonian perspective. In "The Construction of Cognitive Maps", J. Portugali (ed.), 105-132. Kluwer Academic Publishers. Alphen aan den Rijn, Netherlands.

Hilario, M. (1997). An overview of strategies for neurosymbolic integration. In: "Connectionist-Symbolic Integration: From Unified to Hybrid Approaches". R. Sun and F. Alexandre (eds), pgs. 13-36. CRC Press, Boca Raton, FL.

Holmes, N.P. and Spence, C. (2005). Multisensory Integration: space, time and superadditivity. Current Biology, 15, R762-R764.

Hopkins, C.D. (2010). A biological function for electroreception in sharks and rays. Journal of Experimental Biology, 213, 1005-1007.

Hubel, D.H. and Wiesel, T.N. (1962). Receptive fields, binocular interaction and functional architecture in the cat's visual cortex. Journal of Physiology, 160(1), 106-154.

Itti, L. and Baldi, P. (2009). Bayesian surprise attracts human attention. Vision Research, 49(10), 1295-1306.

Johansson, G. (1973). Visual perception of biological motion and a model for its analysis. Perception and Psychophysics, 14(2), 201-211.

Kanai, R., Chang, A., Yu, Y., Magrans de Abril, I., Biehl, M., and Guttenberg, N. (2019). Information generation as a functional basis of consciousness. Neuroscience of Consciousness, 2019(1), niz016.

Kegeleirs, M., Grisetti, G., and Birattari, M. (2021). Swarm SLAM: Challenges and Perspectives. Frontiers in Robotics and Al, 8, 618268. 
Kennedy, J. and Eberhart, R. (2001). Swarm Intelligence. Morgan Kaufmann Publishers, San Francisco, CA.

Kleim, J.A. and Jones, T.A. (2008). Principles of Experience-Dependent Neural Plasticity: Implications for Rehabilitation After Brain Damage. Journal of Speech, Language, and Hearing Research, 51, S225-S239.

Konig, S.U., Schumann, F., Keyser, J., Goeke, C., Krause, C., Wache, S., Lytochkin, A., Ebert, M., Brunsch, V., Wahn, B., Kaspar, K., Nagel, S.K., Meilinger, T., Bulthoff, H., Wolbers, T., Buchel, C., and Konig, P. (2016). Learning New Sensorimotor Contingencies: Effects of Long-Term Use of Sensory Augmentation on the Brain and Conscious Perception. PLoS One, 11(12), e0166647.

Krol, J., Schumann, A., and Bielas, K. (2021). Categorical Approach to Swarm Computations. Proceedings of the International Joint Conference on Biomedical Engineering Systems and Technologies, 14(3), 218-224.

Lafon, G., Howard, S.R., Paffhausen, B.H., Avarguès-Weber, A., and Giurfa, M. (2021). Motion cues from the background influence associative color learning of honey bees in a virtual-reality scenario. Scientific Reports, 11, 21127.

Lan, G. and Tu, Y. (2016). Information Processing in Bacteria: Memory, Computation, and Statistical Physics: a Key Issues Review. Reports on Progress in Physics, 79(5), 052601.

Landauer, R. (1961). Irreversibility and Heat Generation in the Computing Process. IBM Journal of Research and Development, 5(3), 183-191.

Lobo, L., Heras-Escribano, M., and Travieso, D. (2018). The History and Philosophy of Ecological Psychology. Frontiers in Psychology, 9, 2228.

Madiman, M., Johnson, O., and Kontoyiannis, I. (2007). Fisher Information, Compound Poisson Approximation, and the Poisson Channel. IEEE International Symposium on Information Theory, 976-980.

Maruyama, N., Saito, D., Hashimoto, Y., and Ikegami, T. (2019). Dynamic organization of flocking behaviors in a large-scale boids model. Journal of Computational Social Science, 2, 77-84. 
Oizumi, M., Albantakis, L., and Tononi, G. (2014). From Phenomenology to the Mechanisms of Consciousness: Integrated Information Theory 3.0. PLOS Computational Biology, 10(5), e1003588.

Pulliam, H.R. (1973). On the advantages of flocking. Journal of Theoretical Biology, 38(2), 419-422.

Qian, H. and Beard, D.A. (2006). Metabolic futile cycles and their functions: a systems analysis of energy and control. Systems Biology, 153(4), 192-200.

Read, C. and Szokolszky, A. (2018). An Emerging Developmental Ecological Psychology: Future Directions and Potentials. Ecological Psychology, 30, 174-194.

Reina, A., Bose, T., Trianni, V., and Marshall, J.A.R. (2018). Psychophysical Laws and the Superorganism. Scientific Reports, 8, 4387.

Rietveld, E. and Kiverstein, J. (2014). A Rich Landscape of Affordances. Ecological Psychology, 26(4), 325-352.

Reynolds, C.W. (1987). Flocks, Herds, and Schools: a distributed behavioral model. Computer Graphics, 21(4), 25-34.

Ross, B.C. (2014). Mutual Information between Discrete and Continuous Data Sets. PLoS One, 9(2), e87357.

Shannon, C., Weaver, W., and Weiner, N. (1949). The Mathematical Theory of Communication. University of Illinois Press, Champaign-Urbana, IL.

Spivak, D.I. (2014). Category Theory for the Sciences. MIT Press, Cambridge, MA.

Swenson, R. and Turvey, M.T. (1991). Thermodynamic Reasons for Perception -- Action Cycles. Ecological Psychology, 3(4), 317-348.

Tkacik, G. and Gregor, T. (2021). The many bits of positional information. Development, 148(2), dev176065.

Toner, J. and Tu, Y. (1998). Flocks, Herds, and Schools: a quantitative theory of flocking. Physical Review E, 58(4), 4828-4858. 
Turvey, M.T. (2012). From Physical Education to Physical Intelligence: 50 years of Perception-Action. Avant: Trends in Interdisciplinary Studies, 3(2), 128-138.

van Dijk, L., Withagen, R., Bongers, R.M. (2015). Information without content: A Gibsonian reply to enactivist worries. Cognition, 134, 210-214.

Ward, C.R., Gobet, F., and Kendall, G. (2001). Evolving Collective Behavior in an Artificial Ecology. Artificial Life, 7. 191-209.

Weems, S. and Reggia, J. (2012). Behavioral Capacity Limits. Encyclopedia of the Sciences of Learning, doi:10.1007/978-1-4419-1428-6_608.

Wilga, C.D. and Lauder, G.V. (2004). Biomechanics of Locomotion in Sharks, Rays, and Chimeras. In "Biology of Sharks and Their Relatives". D.H. Evans, J.C. Carrier, J.A. Musick, and M.R. Heithaus eds. CRC Press, Boca Raton, FL.

Woods, D.D. (1984). Visual momentum: a concept to improve the cognitive coupling of person and computer. International Journal of Man-Machine Studies, 21, 229-244.

Wu, X., Wang, A., Tang, X., and Zhang, M. (2019). Different visual and auditory latencies affect cross-modal non-spatial repetition inhibition. Acta Psychologica, 200, 102940.

Yu, Y., Ji, H., Wang, L., and Jiang, Y. (2020). Cross-modal social attention triggered by biological motion cues. Journal of Vision, 20(10), 21.

Zhang, T., Rosenberg, M., Perona, P., and Meister, M. (2021). Endotaxis: A Universal Algorithm for Mapping, Goal-Learning, and Navigation. bioRxiv, doi:10.1101/2021.09.24.461751.

Zhou, H., Hu, T., Xie, H., Zhang, D., and Shen, L. (2010). Computational hydrodynamics and statistical modeling on biologically inspired undulating robotic fins: A two-dimensional study. Journal of Bionic Engineering, 7, 66-76. 